\title{
The Influence Of Deviation Of The Geometry Of The Model On The Accuracy Of Researching Loads From Wind Pressure In Wind Tunnels
}

\author{
Oleg Egorychev ${ }^{1, *}$, Vladislav Nosyrin ${ }^{1}$, Marina Radnaeva $^{1}$, and Anastasia Fedosova ${ }^{1}$ \\ ${ }^{1}$ Moscow State University of Civil Engineering, Yaroslavskoe shosse, 26, Moscow, 129337, Russia
}

\begin{abstract}
The construction of unique buildings and structures is widely developing each year; therefore, more attention is paid to investigations of wind influences. The subject of this article describes the effect of deviation in the model geometry of analyzed object on the accuracy of test code on loads from wind pressure in wind tunnel. Four similar models with different geometric shapes are introduced in this article as example, and the results of the test are analyzed below.
\end{abstract}

\section{Introduction}

In connection with wide development of the construction of unique buildings and structures, the designers have a serious question about taking into account the influence of various loads in the design [10]. The main loads and impacts considered in the existing normative documents are climatic (wind, snow), special loads (seismic, explosive), etc. [1, 2]. This loads can be determined according to the current SP 20.13330.2016, nevertheless, aerodynamic coefficients should be assumed on the basis of the test results of the corresponding models in wind tunnels due to the limited number of primitives listed in its annexes, for unique buildings, as well as for buildings with an increased level of responsibility $[1,4]$. The document that classifies the height and uniqueness of building structures is the Town-Planning Code of the Russian Federation (Chapter 6, art. 48.1 "Highly dangerous, technically complex and unique objects"), and also SP 20.13330.2011 "Loads and impacts" (updated version of SNiP 2.01.07-85*).

\section{Description of the models}

One of the most labor-consuming stages is the production of models. There are four models with different geometric shapes introduced in this article: cylinder and prism of square, hexagonal and dodecagonal sections. The checking model, in this case, is a cylinder, since its geometric shape is as close to cylindrical structures and buildings such as vertical tank, silo, bunker, etc. The remaining models are supposed as approximation with different accuracy of geometric similarity.

\footnotetext{
* Corresponding author: olegolege92@gmail.com
} 
In the classical aerodynamic experiment, it is very important to provide for the scale of the reconstituted model, which depends on the size of the working area of the aerodynamic installation, the location of the measuring equipment, and also the method for determining the load from wind pressure.

According to the methods for calculating the wind load, there are two methods of searching the aerodynamic coefficients, as well as the installation of measuring equipment in the model:

1. To determine the aerodynamic coefficient $c_{p}$ at control points. The model assumes the placement in it a measuring system, which includes pressure sensors, pressure taps, and a pneumatic track connecting them;

2. To determine the integral aerodynamic coefficients. The model is mounted on the sensor, which determines the force-moment characteristics [3].

In the case considered in this article, calculation is carried out according to the second method, due to the complexity of erecting pressure sensors in the model. Figure 1 demonstrates the plant layout of models in the wind tunnel, indicating the sensor, the direction of the wind flow and the points of the angle of rotation.

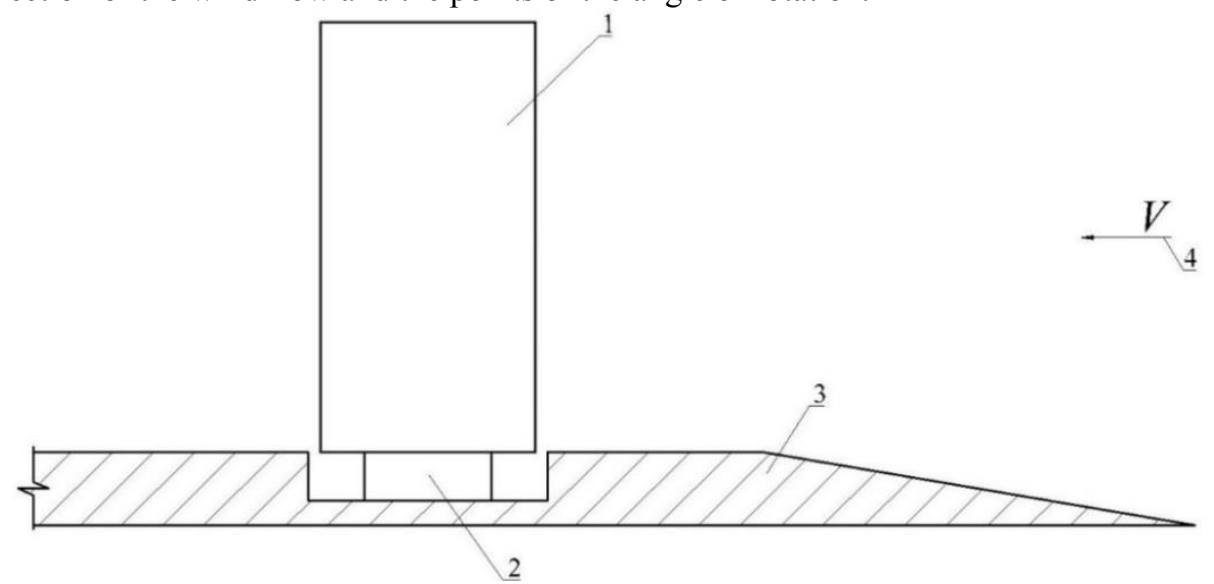

Fig. 1(a). Plant layout of model in the wind tunnel, side view.

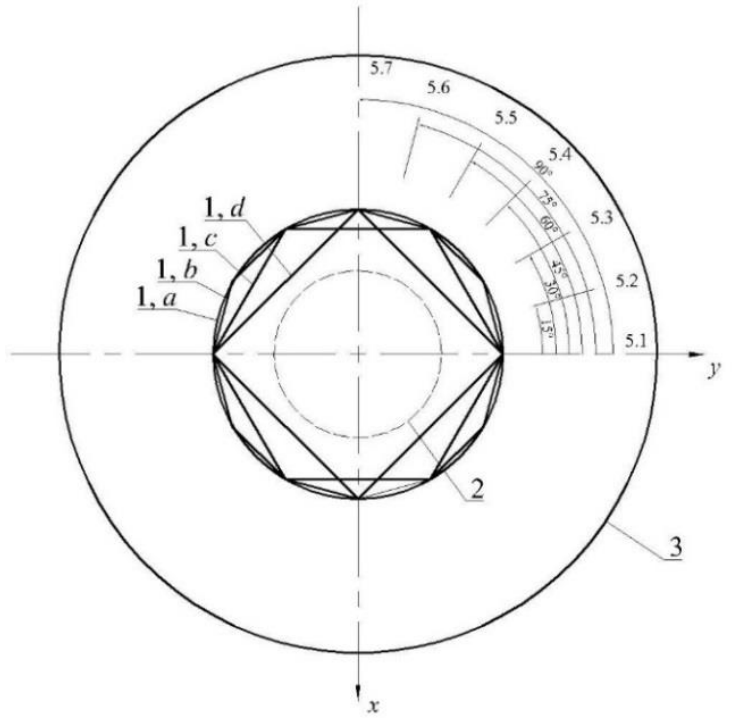

Fig. 1(b). Plant layout of model in the wind tunnel, vertical view. 1- model (1, a - cylinder; $1, b-$ the prism of the twelve-cornered section; 1, c- hexagonal prism; $1, \mathrm{~d}$ - square prism); 2 - sensor, which 
determines the force-moment characteristics; 3 - platform to exclude the impact of wind flow on the sensor; 4-wind flow direction; 5.1-5.7 - points of the angle of rotation during the experiment.

\section{Conduction and results of tests}

When preparation process for running of experiment has been completed velocity of flow is set in the tunnel and data logging protocol is turned on. Rotation of models in the tunnel is carried out in steps of $15^{\circ}$. For each angle sensor data reading is repeated accordingly.

Based on the results of the experiments of determining the force-moment characteristics, the aerodynamic coefficients are calculated by the following formulas:

$$
\begin{gathered}
c_{x}=\frac{F_{x}}{0.5 \cdot \rho \cdot v^{2} \cdot S} ; c_{y}=\frac{F_{y}}{0.5 \cdot \rho \cdot v^{2} \cdot S} ; c_{z}=\frac{F_{z}}{0.5 \cdot \rho \cdot v^{2} \cdot S} \\
c_{m x}=\frac{M_{x}}{0.5 \cdot \rho \cdot v^{2} \cdot S l} ; c_{m y}=\frac{M_{y}}{0.5 \cdot \rho \cdot v^{2} \cdot S l} ; c_{m z}=\frac{M_{z}}{0.5 \cdot \rho \cdot v^{2} \cdot S l}
\end{gathered}
$$

$c_{x}$ - head drag coefficient; $c_{y}$ - side force coefficient; $c_{m z}-$ torque coefficient; $\rho$ - air density; $v$ - velocity of wind flow; $S$ - characteristic area of maximum mid-section; $l-$ moment arm in defined coordinate axes.

After experiment has been conducted only integral coefficients are taken into account $\left(F_{x} ; F_{y} ; M_{z}\right)$, because the remaining coefficients $\left(F_{z} ; M_{x} ; M_{y}\right)$ do not make physical sense in this case.

Having completed the experiment and the transition from the measured values to the aerodynamic coefficients, the obtained values are fixed in tabular and graphical forms in dependence of the coefficient value from the angle of rotation of the model (angle of the wind flow attack).

Table 1 demonstrates the values of the aerodynamic coefficients as a function of the angle of the wind flow attack, as well as the difference in the coefficients $\Delta c_{x}$ and $\Delta c_{y}$ of the prismatic section with the corresponding values for the cylinder.

\section{Table 1.}

\begin{tabular}{|l|c|c|c|c|c|c|}
\hline \multicolumn{1}{|c|}{ Model } & Angle & $c_{x}$ & $c_{y}$ & $c_{m z}$ & $\Delta c_{x}$ & $\Delta c_{y}$ \\
\hline \multirow{2}{*}{ Prism of square section } & 0 & 0,01 & $-0,73$ & $-0,083$ & $-0,0123$ & 0,1123 \\
\cline { 2 - 7 } & 45 & $-0,672$ & $-0,069$ & $-0,001$ & $-0,0916$ & 0,5386 \\
\hline \multirow{2}{*}{ Hexagonal prism } & 0 & 0,044 & $-0,817$ & $-0,112$ & 0,022 & 0,0255 \\
\cline { 2 - 7 } & 45 & $-0,547$ & $-0,74$ & $-0,197$ & 0,034 & $-0,1324$ \\
\hline \multirow{2}{*}{$\begin{array}{l}\text { The prism of the } \\
\text { twelve-sided section }\end{array}$} & 0 & 0,028 & $-0,834$ & $-0,017$ & 0,0056 & 0,0081 \\
\hline \multirow{2}{*}{ Cylinder } & 45 & $-0,58$ & $-0,607$ & $-0,03$ & 0,0005 & $-0,0001$ \\
\cline { 2 - 7 } & 0 & 0,022 & $-0,842$ & $-0,01$ & - & - \\
\cline { 2 - 7 } & 45 & $-0,581$ & $-0,607$ & $-0,025$ & - & - \\
\hline
\end{tabular}

Dependency of the aerodynamic coefficient from the angle of rotation are also demonstrated in diagrams: 


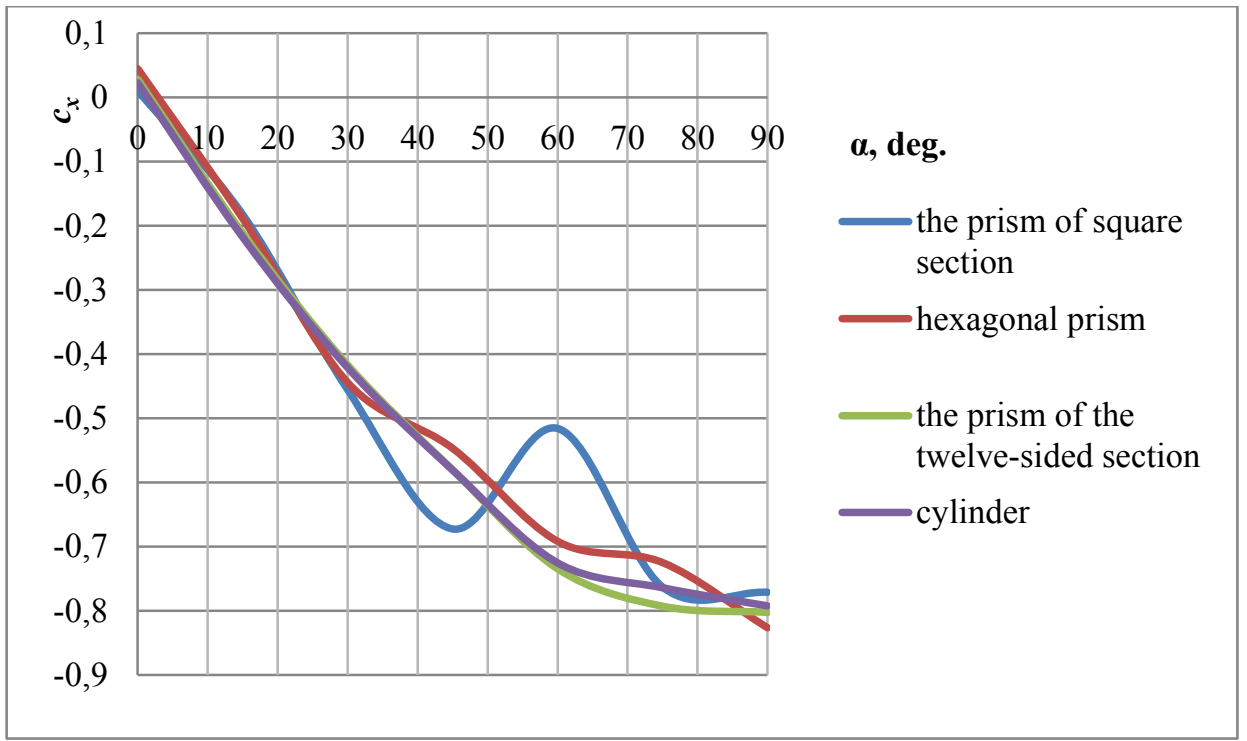

Fig. 2. Dependency of head drag coefficient from angle of the wind flow attack.

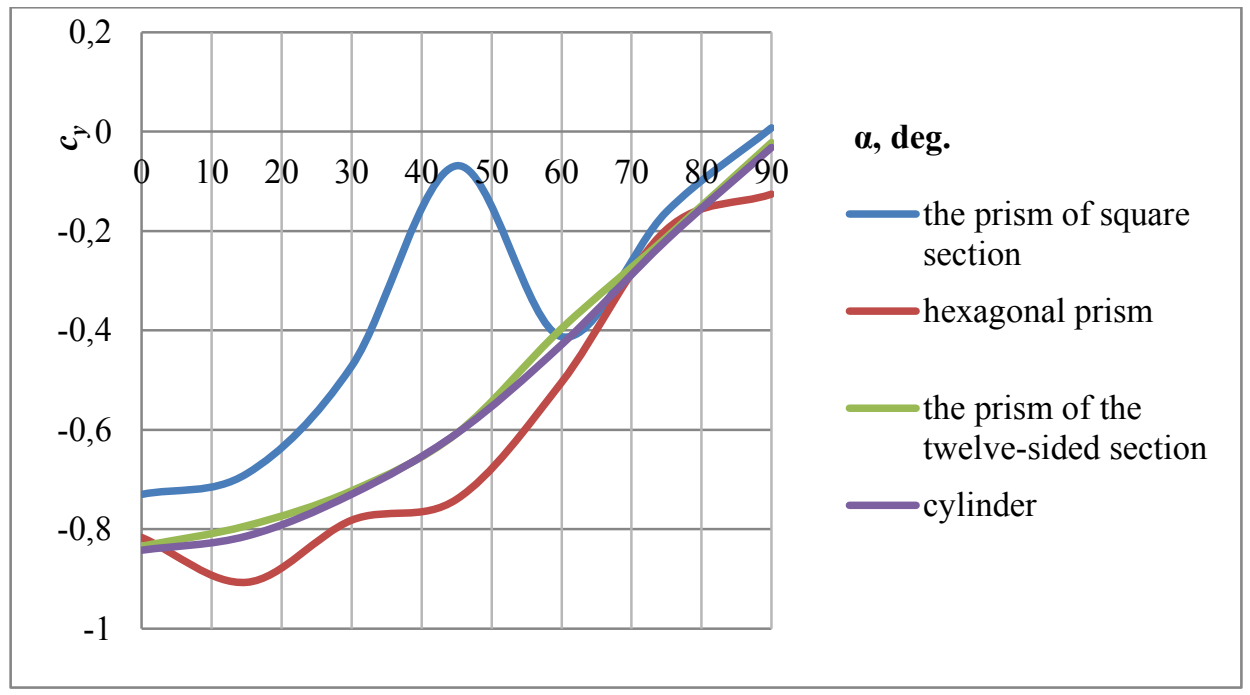

Fig. 3. Dependency of side force coefficient from angle of the wind flow attack 


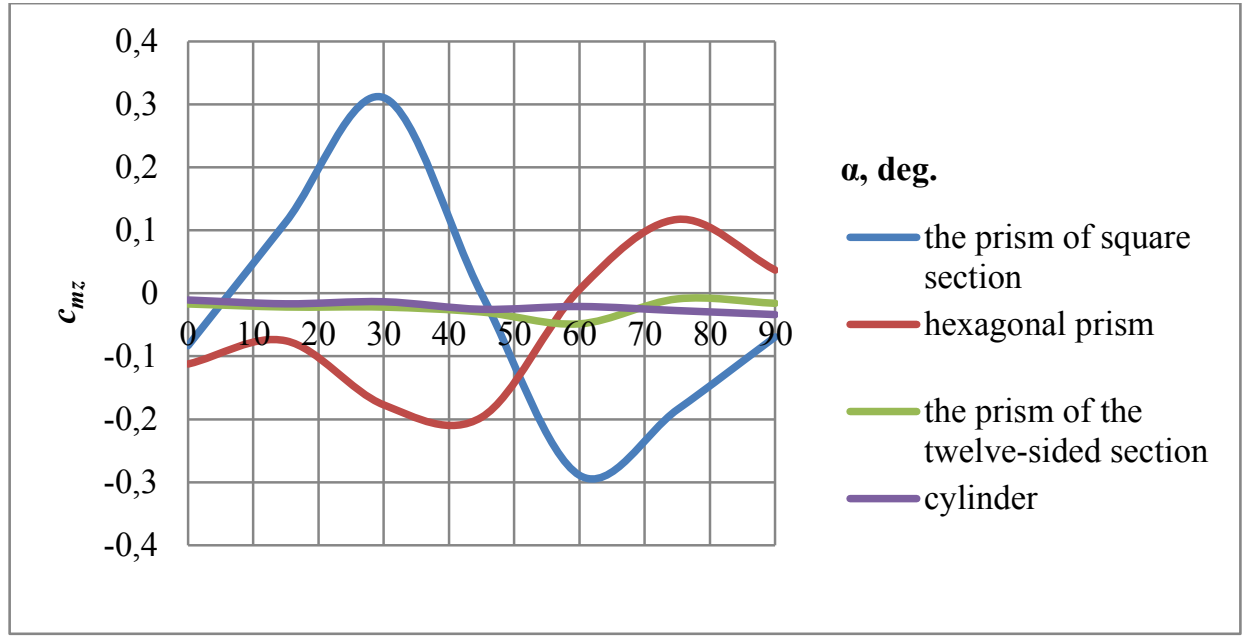

Fig. 4. Dependency of torque coefficient from angle of the wind flow attack

A coefficient that demonstrates how close the tested model is to the checking model (to the cylinder) is input in this case as coefficient $h$ :

$$
h=\frac{a}{A},
$$

$a$ - the length of the perpendicular lowered from the middle to the polygon side to circle, $A$ - diameter of a circle (Figure 5).
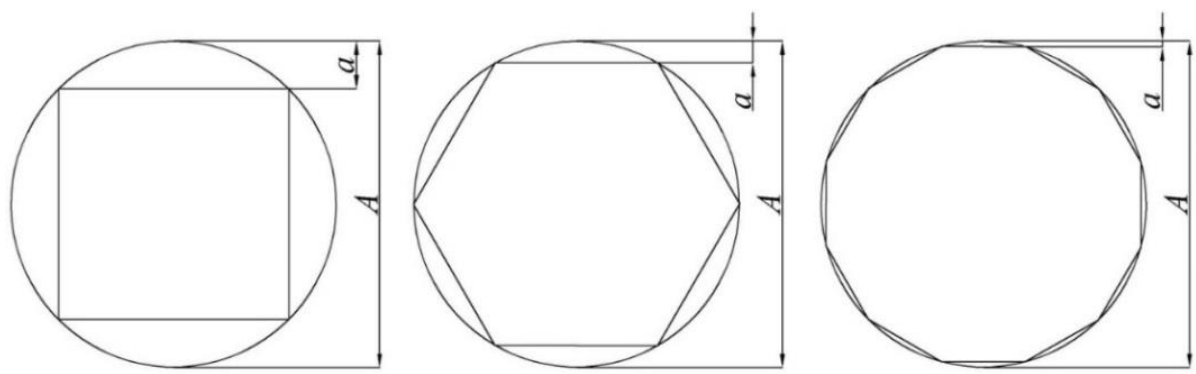

Fig. 5. The polygon inscribed in a circle with lengths designation of $a$ and $A$ in order to input coefficient $h$

The calculation of coefficient hi for each polygon is performed below (there $\mathrm{i}-$ is the number of angles of the polygon):

$$
\begin{aligned}
h_{4}=\frac{0,01025}{0,07} \cong 0,146 ; h_{6}=\frac{0,00469}{0,07} \cong 0,067 ; \\
h_{12}=\frac{0,001193}{0,07} \cong 0,017 ;
\end{aligned}
$$

$h_{4}$ - coefficient for inscribed square; $h_{6}$ - coefficient for inscribed hexagon;

$$
h_{12}-\text { coefficient for inscribed dodecagon. }
$$

Consider the graphs of the dependence of aerodynamic coefficients on the angle of rotation (Figures 2-4). It is clearly illustrated, that the values of the cylinder and the prism of the dodecagonal section are quite close, unlike the other models, since their shape is geometrically closer to each other, and the coefficient $h_{12}$ is rather small. 
It is also clearly shown, that the values of the remaining two prisms significantly differ from the values of the cylinder, and the coefficients $h_{4}$ and $\mathrm{h} 6$ are much greater than $h_{12}$.

Concluding the analysis the following can be stated, the cylindrical design can be modeled by prisms with a section of a dodecagon or with a large number of angles, taking the deviation into account, and the value of coefficient should be $h \leq 0,017$.

Geometric forms where $h>0,02$ are not recommended for usage due to the loss of experiment accuracy.

\section{Conclusions}

It can be noted in general that the geometry of the model significantly affects the accuracy of the research in the wind tunnel. Therefore, during the aerodynamic testing it is very important to create a sufficiently accurate model for the most correct experiment result.

The work was financial supported by the Ministry of Education and Science of the Russian Federation within the framework of the state $\# 7.6075 .2017 / 8.9$, Project «Investigation of the phenomena of aerodynamic instability of building structures in aero-elastic statement, including the development of an innovative methodology for analysing meteorological data to refine the parameters of the wind load».

All tests were carried out using research equipment of the unique scientific installation «Large Research Gradient Wind Tunnel» of The Head Regional Shared Research Facilities of the Moscow State University of Civil Engineering.

\section{References}

1. O. Poddaeva, Y. Buslaeva, D. Gribach, Bulletin of the Belgorod Belgorod State Technological University n.a. V. G. Shukhov, 6, 58-62 (2014)

2. S. Gouvernyuk, O. Egorychev, S. Isaev, N. Kornev, O. Poddaeva, Bulletin of Moscow State University of Civil Engineering, 3, 185-191 (2011)

3. V. Pomelov, D. Gribach, P. Churin, Scientific and Technical Journal Building and Reconstruction, 3, 56 (2016)

4. O. Poddaeva, J. Buslaeva, D. Gribach, Adv Mat Res, 1082, 246-249, (2015)

5. SP 20.13330.2011. Loads and impacts, 80, (2011)

6. Urban Development Code of the Russian Federation of 29.12.2004, (2004)

7. E. Retter, S. Strizhenov, Aerodynamics of buildings (Moscow, Stroiizdat, 240, 1968)

8. E. Retter, Architectural and construction aerodynamics (Moscow, Stroiizdat, 294, 1984)

9. O. Poddaeva, A. Kubenin, P. Churin, M., NRU MGSU, 86, (2017)

10. T. Argentini, D. Rocchi, A. Zasso, J Wind Eng Ind Aerod, 147 (2015)

11. A. Pustovgar, I. Garanzha, L. Shilova, A. Tanasoglo, A. Adamtsevich, V. Ivanilov, Procedia Engineering, 153, 569 (2016) 